\title{
Underdiagnosis and Referral Bias of Autism in Ethnic Minorities
}

\author{
Sander Begeer · Saloua El Bouk · Wafaa Boussaid · \\ Mark Meerum Terwogt $\cdot$ Hans M. Koot
}

Published online: 4 July 2008

(C) The Author(s) 2008

\begin{abstract}
This study examined (1) the distribution of ethnic minorities among children referred to autism institutions and (2) referral bias in pediatric assessment of autism in ethnic minorities. It showed that compared to the known community prevalence, ethnic minorities were under-represented among 712 children referred to autism institutions. In addition, pediatricians $(n=81)$ more often referred to autism when judging clinical vignettes of European majority cases (Dutch) than vignettes including non-European minority cases (Moroccan or Turkish). However, when asked explicitly for ratings of the probability of autism, the effect of ethnic background on autism diagnosis disappeared. We conclude that the use of structured ratings may decrease the likelihood of ethnic bias in diagnostic decisions of autism.
\end{abstract}

Keywords Autism - Developmental assessment .

Diagnostic errors $\cdot$ Stereotyping

\section{Introduction}

Ethnicity and socio-economic status do not consistently influence the prevalence of autism (Bertrand et al. 2001; Croen et al. 2002; Dyches et al. 2004; Palmer et al. 2005; Powell et al. 2000). Still, children from ethnic minority groups seem under-represented in mental health institutions that focus on the treatment of autism (CDC 2006;

\footnotetext{
S. Begeer $(\bowtie) \cdot$ S. E. Bouk · W. Boussaid · M. M. Terwogt · H. M. Koot

Department of Developmental Psychology, VU University Amsterdam, Van der Boechorststraat 1, 1081 Amsterdam, The Netherlands

e-mail: S.Begeer@psy.vu.nl
}

Mandell et al. 2002). Besides ascribing this under-representation to characteristics of ethnic minority groups such as their socio-economic position, different need for special care or parental detection of problems (Mandell and Novak 2005; Thomas et al. 2007), a substantial part of the problem may be attributed to biased professional perceptions of children from ethnic minority groups (Kreps 2006; Mandell and Novak 2005; van Ryn and Fu 2003; Zwirs et al. 2006a).

It has been speculated that professionals screen for autism spectrum disorders (ASD) less often in children from minority than majority groups (Mandell et al. 2002). Language and social problems related to immigrant and ethnic status may hamper proper detection of autistic features. For example, communicative problems that characterize ASD could be unduly interpreted as language problems related to minority groups in general. In addition, problems in the social domain that are characteristic of ASD may in children from immigrant ethnic minority groups be interpreted as problems related to adaptation to the host culture (Reijneveld et al. 2005). Therefore, screening for ASD in ethnic minority groups is complicated by an overlap between social and communicative problem domains that could be attributed to ASD, but also to an ethnic minority background. This could cause underdiagnosis of ASD in ethnic minority groups (Kreps 2006; Mandell et al. 2002; Mandell and Novak 2005; van Ryn and Fu 2003).

One earlier study did not confirm these suggestions in a direct examination of ethnic bias in the diagnostic assessment of ASD. When school psychologists and child psychiatrists were asked to rate clinical case descriptions on the likelihood of various explicitly described diagnostic categories, including ASD, they reported equal probabilities of ASD in minority (African-American) 
or majority (Caucasian) children (Cuccaro et al. 1996). This suggests that bias in diagnostic assessment is not very likely when explicit diagnostic categories are presented.

However, bias in general diagnostic assessment is more likely during spontaneous clinical judgments than following ratings of explicit diagnostic categories (Aklin and Turner 2006; Zalsman et al. 2001). This is alarming since physicians rely on clinical judgment or subjective questions more than on explicit standardized measures (Dosreis and Weiner 2006; King and Glascoe 2003; Sices et al. 2004), despite the recommended use of explicit screening instruments for developmental disorders in infants and young children (Duby et al. 2006). Pediatricians have an important screening function at this early stage (Brugman et al. 2001), and may be biased in their spontaneous assessments, but not when they use explicit diagnostic categories in their assessment procedure.

Though minority groups may be generally under-represented in mental health care (Snowden and Yamada 2005), it is unclear which specific minority groups are underrepresented in specialized ASD health care centers. Studies in the USA have suggested under-diagnosis of ASD in Hispanic children compared to white and black nonHispanic children (CDC 2006), but no differences were found between black and white children (CDC 2006, 2007; Mandell and Novak 2005). In Europe, the under-diagnosis of autism in ethnic minorities has not been studied before, but recent findings suggest different treatment thresholds for non-Western children with behavioural problems, in particular Turkish and Moroccan children (Zwirs et al. 2006b).

In the current study, we first examined whether children from ethnic minorities are under-represented in Dutch ASD institutions (Study 1). Second, we tested whether childrens' ethnic background influenced pediatricians' perceptions of ASD more in spontaneous clinical judgments than in ratings of explicit diagnostic categories (Study 2). The study focused on a comparison of children from the Dutch native majority versus children from Moroccan and Turkish origin, since these constitute the largest non-European minority groups in the Netherlands, representing $4 \%$ of the general population (National Institute for Public Health and Environment 2005).

First, we examined whether the number of children from ethnic minority groups referred to ASD institutions in the Netherlands is indeed lower than might be expected based on the ethnic minorities rates in the general population. This part of the study was conducted by investigating the ethnic background of children in three ASD assessment institutions in the Netherlands, and comparing the proportion of ethnic minority children to the proportion that might be expected based on the representation of minority groups in the general population.
Second, we used a vignette study to investigate whether ethnic background influences the likelihood of pediatricians' references to ASD when using clinical judgments versus ratings of explicit diagnostic categories. Case vignettes were used describing children from the Dutch majority, and European (English, French) and non-European (Turkish, Moroccan) minorities. We expected to find more references to ASD in the spontaneous clinical judgments of majority vignettes, compared to the non-European minority vignettes. No effect of ethnic background was expected following the structured ratings.

\section{Study 1: Distribution of Ethnic Minorities Among Children in ASD Institutions}

Method

\section{Participants}

The distribution of ethnic minority groups was investigated in 712 children and adolescents that were referred to three mental health institutions specialized in ASD, based in the west and east provinces of the Netherlands. These institutions include the two largest ASD assessment centers in the Netherlands and one smaller center. Together, they perform nearly half of all diagnostic assessments of ASD in the Netherlands. The study was based on the case-records of children and adolescents that were referred for diagnostic assessment of ASD in 2003 and 2004. All case records from the small institution were screened $(n=151)$. From the two larger institutions, every fifth case record was screened ( $n=182$ and 379, respectively), representing a total of 2,805 children. Table 1 represents characteristics of the sample.

The sample included $83 \%$ males, which is equivalent to the male ratio reported in autism spectrum samples elsewhere (Yeargin-Allsopp et al. 2003). Furthermore, the distribution of classical autism, Asperger syndrome and PDD-NOS was similar to other reports from Dutch mental health care (van der Gaag and Verhulst 1996).

\section{Scoring}

The observed number of Moroccan and Turkish children was determined by counting the number of referred children with at least one parent born in Morocco or Turkey. The parental place of birth was obtained from the case records. This actual number of children was compared to the expected number of children, calculated based on the minority rates in the birth provinces of all referred children. The yearly minority rates for children in each Dutch province are publicly available from the Dutch Central 
Table 1 Characteristics of 712 children and adolescents referred to autism institutions

\begin{tabular}{|c|c|c|c|c|c|}
\hline Characteristic $^{\mathrm{a}}$ & & $\begin{array}{l}\text { Classical } \\
\text { autism }(20 \%)\end{array}$ & $\begin{array}{l}\text { Asperger } \\
\text { syndrome }(20 \%)\end{array}$ & $\begin{array}{l}\text { PDD-NOS } \\
(39 \%)\end{array}$ & $\begin{array}{l}\text { No autism spectrum } \\
\text { disorder }(21 \%)\end{array}$ \\
\hline Age in years & $13.56(8.81)$ & $6.79(3.93)$ & $9.46(2.74)$ & $9.34(3.34)$ & $7.26(2.84)$ \\
\hline Male $(\%)$ & 83 & 88 & 95 & 86 & 74 \\
\hline \multicolumn{6}{|l|}{ Ethnic background } \\
\hline Dutch $(\%)$ & 76 & 73 & 81 & 78 & 71 \\
\hline Moroccan (\%) & 2 & 2 & 0 & 4 & 2 \\
\hline Turkish (\%) & 2 & 2 & 2 & 0 & 4 \\
\hline Surinam $(\%)$ & 5 & 5 & 4 & 6 & 4 \\
\hline Other European (\%) & 6 & 6 & 4 & 7 & 7 \\
\hline Other non-European (\%) & 9 & 13 & 11 & 6 & 12 \\
\hline
\end{tabular}

${ }^{a}$ Diagnostic information was obtained for $46 \%$ of all children

Bureau of Statistics (www.cbs.nl). To calculate the expected number of Moroccan and Turkish children in the ASD institutions, the actual number of children that were referred to the institutions were multiplied by the minority rates in their province of origin (obtained from the case records). For example, when the actual sample included 100 children from the province of Utrecht, and the rate of Turkish and Moroccan children in Utrecht is $3 \%$, we multiplied 100 with .03 , which comes to three children. These numbers were calculated for all 12 provinces of the Netherlands, and added up to calculate the expected minority rate for a weighted sample of children from the general Dutch population.

Results

The expected number of Moroccan and Turkish children in the weighted sample $(n=712)$ of the general population was $30.5(4.4 \%)$, which is significantly higher than the 15 $(2.1 \%)$ Moroccan and Turkish children that were actually found in the institutions, $p<.05$, Mann-Whitney $U$-test. This finding confirms the expected under-representation of minority groups in ASD institutions.

\section{Study 2: Pediatricians' Reference to ASD in Judging Ethnic Minority Vignettes}

\section{Method}

\section{Participants}

Participants were 82 pediatricians [14 men (17\%), 68 women $(83 \%)$ ] who returned surveys (response rate $29 \%$ ), recruited through the Dutch Physician Child Healthcare Society (Artsen Jeugdgezondheidszorg Nederland), a union of physicians working in child healthcare, representing
1,200 physicians ( $85 \%$ women). One participant failed to fill out the full questionnaire and was not included in the analyses. On average the pediatricians had 17.4 ( $S D=$ 8.5) years of work experience, which is representative for the relatively high age of pediatricians in the Netherlands. Five pediatricians $(6 \%)$ belonged to ethnic minority groups themselves. Pediatric caseloads covered an average annual number of $775.6(S D=596.5)$ children. Of the children constituting these caseloads $15 \%$ were reported to be from Moroccan or Turkish origin. Respondents estimated that $6 \%$ of their overall caseload would ultimately be diagnosed with ASD.

\section{Material}

Six case vignettes were developed to provide descriptive summaries of typical cases that might be encountered by professionals who work with young children. The vignettes were written and reviewed in collaboration with child psychologists and psychiatrists working with children with ASD. Each vignette described the age, developmental level, family situation and daily problems of a school aged boy. Vignettes can be obtained from the first author.

The six vignettes differed in ethnic background, and included two Dutch majority cases, two European minority cases (English or French) and two non-European minority cases (Moroccan or Turkish). Ethnic background was varied independent of the rest of the vignette. Besides the ethnic background, every two vignettes included one, two or three autism features. These features were based on the three main characteristics of autism, as described in the DSM-IV-TR: social impairments, communicative impairments or stereotypical interests and behaviors (APA 2000). Each vignette thus included a unique combination of ethnic background (with two Dutch majority, two European minority and two non-European minority vignettes) and other background information, including the number of 
autistic features (with 2 three feature, 2 two feature and 2 one feature vignettes). This amounted to a $6 \times 6$ Graeco Latin square design (Gardner 1966) with 36 possible vignettes. Each participant received six vignettes, representing a counterbalanced combination of ethnic background and autism features. The vignettes were presented in one of four fixed orders, alternating between ethnic background.

Spontaneous clinical judgments were obtained for each vignette by asking the participants the question "What is the matter with this child?", without any other information. Ratings of explicit diagnostic categories were obtained by presenting participants with explicit diagnostic categories and asking the question "Could you indicate how likely you regard the following disorders for this child?". This question was followed by eight categories: 'Attachment Disorder', 'Neglect', 'Oppositional Defiant Disorder, 'ADHD', 'Autism Spectrum Disorder', 'Language Impairment', 'Learning Disability', 'No Psychopathology', and 'Other'. Each category was followed by five response options, ranging from 'very unlikely' to 'very likely'. The additional diagnostic categories were included to distract respondents' attention from ASD or ethnic minorities.

In addition, respondents were asked how many years they were practicing pediatrics, how many children they saw annually, what percentage of these children were of Dutch, English, French, Turkish or Moroccan origin, and to estimate what percentage of these were eventually diagnosed with ASD.

\section{Procedure}

A packet of materials containing a letter of introduction, a brief demographic sheet, six vignettes, and a stamped return envelope was sent to each potential respondent. Participation in the study by return of the materials was considered to imply informed consent. In a second mailing, respondents were asked specific questions regarding their work experience.

\section{Scoring}

References to ASD in spontaneous clinical judgments. When a clinical judgment included references to autism, autism spectrum disorders, Asperger's syndrome or PDDNOS, this was scored one point. All other responses were scored 0 . Based on these responses proportion scores were calculated over pairs of vignettes that included 1,2 or 3 autism features, and over pairs of vignettes that included Dutch majority, European minority or non-European minority backgrounds.

Likelihood ratings of ASD based on explicit diagnostic categories. Participants were instructed to provide a rating of the likelihood of each diagnostic category on a five-point Likert scale, ranging from 'very unlikely' (1) to 'very likely' (5). Mean likelihood estimates for ASD were computed over vignette pairs that included 1, 2 or 3 autism features (score ranges 1-5), and over vignettes pairs that included Dutch majority, European minority or non-European minority backgrounds (score ranges $1-5$ ).

Results

Effects of the Number of Autistic Features on Spontaneous References and Ratings of Explicit Categories

Because the 36 vignettes could not be evenly distributed over the 81 participants, responses to the vignettes were weighted inversely by the number of times each vignette was presented. Using a Friedman's ANOVA for related samples, it was found that clinical judgments with spontaneous references to ASD systematically increased with the number of autism features included in the vignettes, $\chi^{2}$ $(2)=23.66, p<.001$. The mean proportions of autism references were $.10(S D=.16)$ (one autism feature), .21 $(S D=.22)$ (two autism features) and $.28(S D=.28)$ (three autism features). A similar increase with autism features was found in the likelihood ratings of ASD, $\chi^{2}(2)=61.84$, $p<.001$, with means of $2.56(S D=.78$ ) (one autism feature), 3.28 ( $S D=.67$ ) (two autism features) and 3.54 $(S D=.77)$ (three autism features).

\section{Effect of Ethnic Background on Spontaneous References} and Ratings of Explicit Categories

Friedman's ANOVA for related samples indicated that ethnic background influenced the proportion of clinical judgments with spontaneous references to autism, $\chi^{2}(2)=$ $8.70, p<.05$. The mean proportions were $.23(S D=.25)$ (Dutch majority), .21 ( $S D=.25)$ (European minority), and $.14(S D=.20)$ (non-European minority). Mean ranks were 2.17, 2.01 and 1.83, respectively. Dutch majority vignettes elicited more references to ASD compared to non-European minority vignettes, $Z=-1.91, p<.05$ (Wilcoxon test, onetailed), but not compared to European minority vignettes, $Z=-.06, n s$ (Table 2). In contrast to the clinical judgments, the likelihood ratings of ASD were similar in Dutch majority $($ mean $=3.36, S D=.85)$, European minority (mean $=3.19, S D=.82$ ) and non-European minority $($ mean $=3.08, S D=.85), \chi^{2}(2)=2.36$, ns. Mean ranks were $2.13,1.94,1.93$, respectively (Table 3 ). In addition, similar results were found when the likelihood ratings were recorded as dichotomous variables ("likely" or "very likely" (1) versus all other responses (0)): Dutch majority $($ mean $=.59, S D=.49)$, European minority $($ mean $=.47$, $S D=.50)$ and non-European minority (mean $=.46$, 
Table 2 Mean proportion $(S D)$ of autism references based on clinical judgments

\begin{tabular}{llll}
\hline Ethnic background & $\begin{array}{l}\text { One autism } \\
\text { feature }\end{array}$ & $\begin{array}{l}\text { Two autism } \\
\text { features }\end{array}$ & $\begin{array}{l}\text { Three autism } \\
\text { features }\end{array}$ \\
\hline Dutch majority & $.15(.39)$ & $.21(.44)$ & $.33(.61)$ \\
European minority & $.09(.28)$ & $.27(.55)$ & $.26(.54)$ \\
Non-European minority & $.06(.24)$ & $.14(.34)$ & $.23(.45)$
\end{tabular}

Note: Each proportion represents the mean number of autism references calculated over two vignettes

Table 3 Mean likelihood ratings $(S D)$ of autism spectrum disorders (range 1-5)

\begin{tabular}{llll}
\hline Ethnic background & $\begin{array}{l}\text { One autism } \\
\text { feature }\end{array}$ & $\begin{array}{l}\text { Two autism } \\
\text { features }\end{array}$ & $\begin{array}{l}\text { Three autism } \\
\text { features }\end{array}$ \\
\hline Dutch majority & $2.95(.80)$ & $3.50(.50)$ & $3.81(.37)$ \\
European minority & $2.61(.74)$ & $3.17(.80)$ & $3.70(.72)$ \\
Non-European minority & $2.62(.74)$ & $3.54(.72)$ & $3.67(.43)$ \\
\hline
\end{tabular}

Note: Each proportion represents the likelihood ratings of an autism spectrum disorder ranging from 'very unlikely' (1) to 'very likely' (5) calculated over two vignettes

$S D=.50), \chi^{2}(2)=3.52, n s$, mean ranks $2.13,1.94,1.93$, respectively. Finally, no ordering effects were found on spontaneous references or likelihoods ratings of ASD.

\section{Correlations Between ASD References and Ratings and Work Experience}

Spearman's correlations were used to assess the relation between ASD references or ASD ratings and work experience, experience with ASD, or experience with ethnic minorities. None of these correlations were significant (all $p$ 's <.2). Note that pediatricians estimated $15 \%$ of their caseload to be of Moroccan or Turkish origin, which is substantially higher than the community prevalence rate of $4 \%$. These results may be related to lower SES causing higher psychopathology rates in minority groups (Bruce et al. 1991), and more use of care (Snowden et al. 2008). However, unduly high estimations could also be due to social desirability or illusory correlation (Shavitt et al. 1999).

\section{References and Likelihood Ratings of other Diagnostic Criteria}

Though we made no explicit hypotheses about the references and likelihood ratings of diagnostic criteria besides ASD, we exploratively analyzed these results to provide a fuller overview of the current data. In general, the diagnostic categories that were spontaneously referred to most often were language impairments $(.12, S D=.16)$, and
ADHD (.10, $S D=.13)$. No differences were found between the prevalence of alternative diagnostic categories by ethnic background.

\section{Discussion}

A case record investigation of 712 records representing about 3,000 children with ASD indicated an underdiagnosis of children from ethnic minorities in ASD institutions. This finding is in line with a subtle bias that was found among pediatricians' assessment of ASD in ethnic minority groups. Pediatricians' spontaneous clinical judgments based on Dutch majority vignettes more often included references to ASD than their judgments of non-European minority vignettes. However, when pediatricians were explicitly instructed to rate the likelihood of an autism spectrum disorder, the bias of ethnic background disappeared entirely. This confirms a previous finding that ethnic bias in ASD assessment does not affect screening when explicit diagnostic categories are used in the assessment procedure (Cuccaro et al. 1996).

The bias in spontaneous clinical judgments, which was unrelated to work experience of the pediatricians, may be explained by the superficial similarity of social and communicative problem domains of children with ASD and children from minority groups. Pediatricians may be inclined to attribute social and communicative problems of children from non-European minority groups to their ethnic origin, while they would possibly attribute the same problems to autistic disorders in children from majority groups. The absence of a bias regarding European minority cases suggests that in particular cultural differences or the combination of language and cultural differences seem to affect professional assessments. This indicates that, based on spontaneous clinical judgments, non-European minority groups do no receive the same access to autism diagnoses and treatments in comparison to majority groups.

In the assessment of ASD, the use of standardized questionnaires strongly improves the quality of diagnostic assessment (Risi et al. 2006). Since these questionnaires are unlikely to be administered during the early stages of the assessment procedure, it may be more advantageous to include a simple screening list in procedures used in the general pediatric practice. In the USA, only $8 \%$ of general pediatricians screen for ASD on a regular basis. Most pediatricians report to have insufficient time for specific screening on ASD, but a majority also reports to be unfamiliar with screeners (Dosreis and Weiner 2006). A higher awareness of the danger of possible biases in subjective screening may help increase the use of explicit screening devices in pediatric practice. However, it should be noted that the absence of ethnic bias following structured 
diagnostic ratings in a vignette study does not insure the absence of such bias in clinical practice. The opportunity to estimate the probability of diagnoses may have resulted in demand characteristics, which are a limitation of vignette studies. More research is needed to confirm that the use of structured ratings will reduce pediatricians' ethnic bias in their real life judgments, and whether this effect will prevail over repeated use of such rating procedures.

Several other limitations of the current study have to be noticed. First, a nation wide survey is needed to confirm the suggested under-representation of minority groups in ASD institutions. Second, the pediatrician sample included a majority of females, while most studies show a predominance of male pediatricians (Cull et al. 2002; Dosreis and Weiner 2006). Female pediatricians refer children more often for psychiatric diagnostic assessment than their male colleagues (Sices et al. 2004). However, the current study showed no gender effects. Third, the vignettes were not controlled for SES, and pediatricians may have perceived a possible correlation between SES and ethnicity, thus providing an additional explanation for the nature of the current bias. Finally, specific cultural aspects that play a role in the assessment procedures may vary widely across cultures, and also depend on the ethnic background of the pediatricians themselves. Due to the low number of pediatricians with a non-Dutch ethnicity in the sample we were not able to test this latter possibility. A wider cross-cultural study is needed to take full account of the factors involved in the diagnostic process.

A question that remains unsolved is why the presumed overlap in problem domains of children from ethnic minority groups and children with ASD does not result in overdiagnosis of ASD in ethnic minorities, i.e., false positive diagnoses resulting from social and communicative problems that are misinterpreted as autism features but are in fact due to cultural adaptive and communicative problems. The absence of such findings could suggest that pediatricians adequately separate language problems with Dutch as a second language from neurodevelopmental delay. Still, Western pediatricians may also have a stereotypical view of autism as a 'western' disorder, possibly also related to higher SES (Bhasin and Schendel 2007; Newschaffer et al. 2007). While this view has long been renounced (Volkmar and Pauls 2003), it would be worthwhile to investigate whether these stereotypes are still prevalent among pediatricians.

Acknowledgments We thank Noel van Erp for his assistance in analyzing the data, the participating pediatricians for their time, and De Bascule, Duivendrecht, Centrum Autisme, Oegstgeest, and het Dr. Leo Kannerhuis, Doorwerth, for their kind collaboration.

Open Access This article is distributed under the terms of the Creative Commons Attribution Noncommercial License which permits any noncommercial use, distribution, and reproduction in any medium, provided the original author(s) and source are credited.

\section{References}

Aklin, W. M., \& Turner, S. M. (2006). Toward understanding ethnic and cultural factors in the interviewing process. Psychotherapy, 43, 50-64.

American Psychiatric Association. (2000). Diagnostic and statistical manual of mental disorders (4th ed.). Washington, DC: American Psychiatric Association.

Bertrand, J., Mars, A., Boyle, F., Yeargin-Allsopp, M., \& Decoufle, P. (2001). Prevalence of autism in a United States population: The Brick Township, New Jersey, investigation. Pediatrics, 108, 1155-1161.

Bhasin, T. K., \& Schendel, D. (2007). Sociodemographic risk factors for autism in a US metropolitan area. Journal of Autism and Developmental Disorders, 37, 667-677.

Bruce, M. L., Takeuchi, D. T., \& Leaf, P. J. (1991). Poverty and psychiatric status-longitudinal evidence from the New-Haven epidemiologic catchment-area study. Archives of General Psychiatry, 48, 470-474.

Brugman, E., Reijneveld, S. A., Verhulst, F. C., \& VerlooveVanhorick, S. P. (2001). Identification and management of psychosocial problems by preventive child health care. Archives of Pediatrics and Adolescent Medicine, 155, 462-469.

CDC. (2006). Mental health in the United States: Parental report of diagnosed autism in children aged 4-17 years-United States, 2003-2004. Morbidity and Mortality Weekly Report, 55, 481486.

CDC. (2007). Surveillance summaries: Prevalence of autism spectrum disorders. Morbidity and Mortality Weekly Report, 56, 1-28.

Croen, L. A., Grether, J. K., \& Selvin, S. (2002). Descriptive epidemiology of autism in a California population: Who is at risk? Journal of Autism and Developmental Disorders, 32, 217224.

Cuccaro, M. L., Wright, H. H., Rownd, C. V., Abramson, R. K., Waller, J., \& Fender, D. (1996). Professional perceptions of children with developmental difficulties: The influence of race and socioeconomic status. Journal of Autism and Developmental Disorders, 26, 461-469.

Cull, W. L., Mulvey, H. J., O’Connor, K. G., Sowell, D. R., Berkowitz, C. D., \& Britton, C. V. (2002). Pediatricians working part-time: Past, present, and future. Pediatrics, 109, 1015-1020.

Dosreis, S., \& Weiner, C. L. (2006). Autism spectrum disorder screening and management practices among general pediatric providers. Journal of Developmental and Behavioral Pediatrics, 27, S88-S94.

Duby, J. C., Lipkin, P. H., Macias, M. M., Wegner, L. M., Duncan, P., Hagan, J. F., et al. (2006). Identifying infants and young children with developmental disorders in the medical home: An algorithm for developmental surveillance and screening. Pediatrics, 118, $405-420$.

Dyches, T. T., Wilder, L. K., Sudweeks, R. R., Obiakor, F. E., \& Algozzine, B. (2004). Multicultural issues in autism. Journal of Autism and Developmental Disorders, 34, 211-222.

Gardner, M. (1966). New mathematical diversions. New York: Simon \& Schuster.

King, T. M., \& Glascoe, F. P. (2003). Developmental surveillance of infants and young children in pediatric primary care. Current Opinion in Pediatrics, 15, 624-629.

Kreps, G. L. (2006). Communication and racial inequities in health care. American Behavioral Scientist, 49, 760-774. 
Mandell, D. S., Listerud, J., Levy, S. E., \& Pinto-Martin, J. A. (2002). Race differences in the age at diagnosis among medicaid-eligible children with autism. Journal of the American Academy of Child and Adolescent Psychiatry, 41, 1447-1453.

Mandell, D. S., \& Novak, M. (2005). The role of culture in families' treatment decisions for children with autism spectrum disorders. Mental Retardation and Developmental Disabilities Research Reviews, 11, 110-115.

National Institute for Public Health, Environment. (2005). Nationale Atlas Volksgezondheid. Bilthoven: RIVM.

Newschaffer, C. J., Croen, L. A., Daniels, J., Giarelli, E., Grether, J. K., Levy, S. E., et al. (2007). The epidemiology of autism spectrum disorders. Annual Review of Public Health, 28, 235258.

Palmer, R. F., Blanchard, S., Jean, C. R., \& Mandell, D. S. (2005). School district resources and identification of children with autistic disorder. American Journal of Public Health, 95, 125130.

Powell, J. E., Edwards, A., Edwards, M., Pandit, B. S., SungumPaliwal, S. R., \& Whitehouse, W. (2000). Changes in the incidence of childhood autism and other autistic spectrum disorders in preschool children from two areas of the West Midlands, UK. Developmental Medicine and Child Neurology, 42, 624-628.

Reijneveld, S., Harland, P., Brugman, E., Verhulst, F., \& VerlooveVanhorick, S. (2005). Psychosocial problems among immigrant and non-immigrant children-Ethnicity plays a role in their occurrence and identification. European Child and Adolescent Psychiatry, 14, 145-152.

Risi, S., Lord, C., Gotham, K., Corsello, C., Chrysler, C., Szatmari, P., et al. (2006). Combining information from multiple sources in the diagnosis of autism spectrum disorders. Journal of the American Academy of Child and Adolescent Psychiatry, 45, 1094-1103.

Shavitt, S., Sanbonmatsu, D. M., Smittipatana, S., \& Posavac, S. S. (1999). Broadening the conditions for illusory correlation formation: Implications for judging minority groups. Basic and Applied Social Psychology, 21, 263-279.
Sices, L., Feudtner, C., McLaughlin, J., Drotar, D., \& Williams, M. (2004). How do primary care physicians manage children with possible developmental delays? A national survey with an experimental design. Pediatrics, 113, 274-282.

Snowden, L. R., Masland, M. C., Libby, A. M., Wallace, N., \& Fawley, K. (2008). Racial/ethnic minority children's use of psychiatric emergency care in California's public mental health system. American Journal of Public Health, 98, 118-124.

Snowden, L. R., \& Yamada, A. M. (2005). Cultural differences in access to care. Annual Review of Clinical Psychology, 1, 143166.

Thomas, K. C., Ellis, A. R., McLaurin, C., Daniels, J., \& Morrissey, J. P. (2007). Access to care for autism-related services. Journal of Autism and Developmental Disorders, 37, 1902-1912.

van der Gaag, R. J., \& Verhulst, F. C. (1996). Aan autisme verwante stoornissen: Een inleiding. Kind en Adolescent, 17, 57-61.

van Ryn, M., \& Fu, S. S. (2003). Paved with good intentions: Do public health and human service providers contribute to racial/ ethnic disparities in health? American Journal of Public Health, 93, 248-255.

Volkmar, F. R., \& Pauls, D. (2003). Autism. Lancet, 362, 1133-1141.

Yeargin-Allsopp, M., Rice, C., Karapurkar, T., Doernberg, N., Boyle, C., \& Murphy, C. (2003). Prevalence of autism in a US metropolitan area. Journal of the American Medical Association, $289,49-55$.

Zalsman, G., Horesh, N., Arzi, R., Edelist, D., Even, D. H., Tyano, S., et al. (2001). Psychosocial diagnosis in psychiatrically hospitalized adolescents. Comprehensive Psychiatry, 42, 223-227.

Zwirs, B. W. C., Burger, H., Buitelaar, J. K., \& Schulpen, T. W. J. (2006a). Ethnic differences in parental detection of externalizing disorders. European Child and Adolescent Psychiatry, 15, 418426.

Zwirs, B. W. C., Burger, H., Schulpen, T. W. J., \& Buitelaar, J. K. (2006b). Different treatment thresholds in non-western children with behavioral problems. Journal of the American Academy of Child and Adolescent Psychiatry, 45, 476-483. 